\title{
Peso insuficiente ao nascer: estudo de fatores associados em duas coortes de recém-nascidos em Ribeirão Preto, São Paulo
}

\author{
Insufficient birth weight: factors associated in two cohorts of newborns in Ribeirão Preto, São Paulo, Brazil
}

Veidson Marcelo Azenha' ${ }^{1}$, Melina Andrade Mattar², Viviane Cunha Cardoso ${ }^{3}$, Marco Antonio Barbieri', Luiz Antonio Del Ciampo ${ }^{4}$, Heloisa Bettiol${ }^{4}$

\section{RESUMO}

Objetivo: Avaliar fatores associados ao aumento das taxas de peso insuficiente (PI) em duas coortes de recém-nascidos (RN) vivos e de parto único de Ribeirão Preto, São Paulo, separadas por 15 anos (1978/79 e 1994).

Métodos: Foram estudados 6.223 partos em 1978/79 e 2.522 em 1994, excluindo-se os RN de baixo peso. Associação entre PI e variáveis independentes (idade, trabalho, escolaridade e tabagismo maternos, abortos e natimortos prévios, número de filhos, situação conjugal, visitas de prénatal, tipo de parto e hospital, categoria de internação, renda familiar, idade gestacional e sexo do RN) foi analisada por cálculo da Odds Ratio (OR) bruto e intervalo de confiança 95\%, seguida de regressão logística múltipla.

Resultados: Em 1978/79, na análise ajustada, idade materna $<20$ anos $(p=0,014)$, renda familiar $<5$ saláriosmínimos $(p=0,030)$, menos de quatro consultas de prénatais $(p=0,003)$, parto vaginal $(p<0,001)$, sexo feminino $(p<0,001)$, tabagismo materno $(p<0,001)$ e prematuridade $(p<0,001)$ estiveram associados a PI. Em 1994, os fatores associados ao PI foram: trabalho materno fora do lar $(p=0,020)$, sexo feminino $(p<0,001)$, tabagismo materno $(p<0,001)$ e prematuridade $(p<0,001)$.

Conclusões: Variáveis socioeconômicas que atuaram em 1978/79 desapareceram em 1994, restando o sexo do RN, o hábito de fumar e a prematuridade nos dois períodos. Como houve diminuição do tabagismo materno e não houve modificação na proporção de nascimentos do sexo feminino, uma explicação para o aumento do PI seria o aumento nos nascimentos pré-termo entre as duas coortes.

Palavras-chave: peso insuficiente; recém-nascido; peso ao nascer; prematuro.

\section{ABSTRACT}

Objective: To evaluate factors associated to the increase in insufficient birth weight (IBW) rates in two populationbased-cohorts of singletons born alive in Ribeirão Preto, São Paulo, Brazil, separated by 15 years (1978/79 and 1994).

Methods: The first cohort (1978/79) comprised 6,223 newborns and the second (1994), 2,522, excluding low birth weight newborns. Independent variables association with IBW (maternal age, work, schooling and smoking; previous abortion, previous stillbirth, number of live births, maternal marital status, family income, occupation group, type of hospital, mode of insurance, number of antenatal visits, type of delivery; newborn gender and gestational age) were analyzed. The Odds Ratio (OR) was determined using a logistic regression model and 95\% confidence limits were calculated.

Results: In 1978/79, maternal age $<20$ years $(p=0.014)$, family income $<5$ minimum Brazilian wages $(p=0.030)$, $<4$ antenatal visits $(p=0.003)$, vaginal delivery $(p<0.001)$, female newborn gender $(p<0.001)$, maternal smoking $(p<0.001)$ and preterm birth $(p<0.001)$ were associated with IBW. In 1994, maternal work outside home
${ }^{1}$ Mestre em Pediatria pelo curso de pós-graduação da Faculdade de Medicina de Ribeirão Preto da Universidade de São Paulo (FMRP-USP), Ribeirão Preto, SP, Brasil

${ }^{2}$ Aluna do sexto ano do curso de Ciências Médicas da FMRP-USP e bolsista de Iniciação Científica da Fundação de Amparo à Pesquisa do Estado de São Paulo (Fapesp), Ribeirão Preto, SP, Brasil

${ }^{3}$ Doutoranda do curso de pós-graduação da FMRP-USP, Programa Saúde da Criança e do Adolescente, Ribeirão Preto, SP, Brasil

${ }^{4}$ Docente do Departamento de Puericultura e Pediatria da FMRP-USP, Ribeirão Preto, SP, Brasil
Endereço para correspondência:

Heloisa Bettiol

Departamento de Puericultura e Pediatria da FMRP-USP

Avenida Bandeirantes, 3.900

CEP 14049-900 - Ribeirão Preto/SP

E-mail: hbettiol@fmrp.usp.br

Fontes financiadoras: Conselho Nacional de Desenvolvimento Científico e Tecnológico (CNPq) e Fundação de Amparo à Pesquisa do Estado de São Paulo (Fapesp), processo número 1993/0525-3 e 05/56516-9 
$(p=0.020)$, female newborn gender $(p<0.001)$, maternal smoking $(p<0.001)$ and preterm births $(p<0.001)$ were associated with IBW.

Conclusions: Socioeconomics variables associated with IBW in 1978/79 were not determinants in 1994. Newborn gender, maternal smoking and preterm birth remained significant determinants of IBW in both moments. Considering the decrease in rate of maternal smoking and the maintenance of female births, the increase in IBW could be explained by increasing rates of preterm birth in this interval of 15 years.

Key-words: insufficient birth weight; infant, newborn; birth weight; infant, premature.

\section{Introdução}

O peso ao nascer é reconhecidamente um importante parâmetro relacionado com a morbimortalidade perinatal e infantil e, por isso, de grande relevância em Saúde Pública. Há inúmeras referências de que crianças nascidas de baixo peso (menor que $2.500 \mathrm{~g}$ ) apresentam, com maior freqüência, alterações de crescimento e desenvolvimento ${ }^{(1-3)}$, retardo mental e distúrbios de aprendizado ${ }^{(1)}$, além de o baixo peso contribuir para o desenvolvimento de doenças crônicas não transmissíveis do adulto ${ }^{(4)}$.

Nos últimos anos, alguns estudos têm mostrado que o peso insuficiente (PI, peso entre 2.500 e $2.999 \mathrm{~g}$ ), assim como o baixo peso ao nascer, está associado à maior morbimortalidade infantil e a conseqüências em longo prazo, como as citadas acima ${ }^{(5)}$. Tal fato adquire grande magnitude quando se considera que uma fração expressiva dos recém-nascidos $(\mathrm{RN})$ tem peso situado neste intervalo. Alguns trabalhos mostram que o número de crianças com PI chega a ser duas a três vezes maior que as de baixo peso ${ }^{(1,6,7)}$. Apesar disso, são encontrados poucos estudos sobre o PI ao nascer.

Há mais de 30 anos, Puffer e Serrano ${ }^{(8)}$ já ressaltavam a importância do nascimento com peso favorável $(>3.000 \mathrm{~g}$ ) para a sobrevivência, crescimento e desenvolvimento sadios. Puffer e Serrano ${ }^{(9)}$ estudaram as características do peso ao nascer em diversos países. Em 1973, 11,6\% das crianças nasciam com PI na Suécia. Em Cuba, este percentual era de 26,8\% e, na Hungria, 23,6\%. Na Índia, de 1969 a 1972, 45,8\% apresentavam PI ao nascimento. Nos Estados Unidos, em 1983, 16,1\% dos nascidos tinham PI. Em 1977, 24,9\% das crianças no Chile e $24,2 \%$ no Uruguai nasceram com peso situado neste intervalo.
No Brasil, existem grandes diferenças regionais na distribuição do baixo peso e do PI. Porém, a maioria dos estudos não é representativa da população, mas restrita a hospitais ou dados populacionais parciais ${ }^{5}$. Souza et $a l^{(7)}$, ao estudar o peso de nascidos vivos, de 1978 e 1979, em maternidades de Florianópolis, Santa Catarina, observaram que $15,3 \%$ nasciam com PI. Esse percentual foi de $31 \%$ em uma amostra de $\mathrm{RN}$ de mães de condição socioeconômica baixa em Recife, Pernambuco ${ }^{(1)}$, e de 31,6\% em maternidades do município de São Paulo ${ }^{(10)}$.

$\mathrm{Kramer}^{(11)}$, em extensa revisão da literatura publicada em 1987 , apresenta os fatores de risco para o baixo peso de nascimento mais citados nos trabalhos e os distribui em categorias que, muitas vezes, se relacionam e se superpõem: fatores constitucionais e genéticos, obstétricos e relacionados à atenção prénatal, nutricionais, morbidade materna, fatores relacionados à exposição tóxica, fatores demográficos e socioeconômicos. Acredita-se que esses fatores também possam estar relacionados ao PI, mas sua influência sobre este é pouco estudada.

Em Ribeirão Preto, São Paulo, a comparação entre duas coortes de RN com intervalo de 15 anos, sendo a primeira em 1978/79 e a segunda em 1994, mostrou aumento significativo na proporção de nascimentos de PI, de $22,4 \%$ para $28,7 \%^{(12)}$. Ao mesmo tempo, observou-se aumento da taxa de baixo peso ao nascer e diminuição da média de peso de nascimento, sugerindo que os mesmos fatores que atuaram no aumento da taxa de baixo peso também estariam influenciando o aumento na proporção de PI ao nascer. O objetivo deste trabalho é avaliar os fatores associados ao aumento do PI ao nascer em Ribeirão Preto nesses dois momentos (1978/79 e 1994).

\section{Métodos}

Os dois estudos foram realizados em todos os hospitais de Ribeirão Preto que, em 1978/79 e 1994, possuíam maternidades, nas quais ocorreram mais de $98 \%$ dos nascimentos do município ${ }^{(13)}$. Todos os nascimentos vivos de parto único de famílias residentes no município foram incluídos na análise. Na primeira população estudada, foram coletados dados de 6.750 partos, de junho de 1978 a maio de 1979. Como não houve sazonalidade de nascimentos em 1978/79, o segundo estudo analisou 2.846 nascimentos durante quatro meses, de maio a agosto de $1994^{(8)}$. Um questionário padronizado foi usado e as mães foram entrevistadas logo após o parto. Quando a mãe não era entrevistada, os dados foram obtidos do prontuário médico. Assim, todos os nascimentos foram incluídos, embora em alguns casos os dados estivessem 
incompletos porque a mãe não foi entrevistada. A metodologia utilizada foi a mesma em ambas as pesquisas ${ }^{(13,14)}$.

Os dados coletados ao nascimento foram codificados por pessoal treinado, segundo um manual previamente confeccionado e testado, digitados e armazenados em microcomputador. Foram feitas as conferências da codificação e digitação e processadas as correções necessárias.

As variáveis maternas incluídas nesta análise foram: idade (<20 anos, 20-24, 25-29, 30-34, 35 anos e mais), trabalho fora do lar (não/sim), abortos prévios (não/sim), natimortos prévios (não/sim), número de filhos incluindo atual gravidez (1, 2-4, 5 e mais), tabagismo durante a gestação (não/sim), escolaridade materna ( 4 anos ou menos, 5-8, 9-11, 12 anos ou mais). As variáveis socioeconômicas e relacionadas à gestação e ao parto foram: ocupação do chefe de família (não manual, manual qualificado e semiqualificado, manual não qualificado e desempregado), situação conjugal materna (com e sem companheiro), número de visitas no pré-natal (0, 1-3, 4-5, 6 e mais), tipo de parto (cesárea ou vaginal), categoria de internação para o parto (público, privado), renda familiar mensal em quantidade de salários-mínimos (menor que 3, 3-4, 5-9, 10 e mais), tipo de hospital onde foi realizado o parto: universitário, público (hospitais privados que atendem predominantemente seguro público), privado (hospitais privados que atendem predominantemente seguro privado e/ou pacientes particulares). Relativas ao RN, foram incluídas as seguintes variáveis: idade gestacional, medida em semanas completas pela data da última menstruação (quando ignorada ou implausível, foi feita a imputação da idade gestacional em um modelo de regressão usando-se peso de nascimento), paridade, renda familiar, sexo do $\mathrm{RN}^{(15)}$ e peso de nascimento (2.500-2.999g: PI, e peso $\geq 3.000 \mathrm{~g}$ : peso favorável). As crianças nascidas de baixo peso $(<2.500 \mathrm{~g})$ foram excluídas da análise.

Foi feita análise descritiva por tabelas de freqüência para caracterizar as coortes de crianças nascidas nos dois períodos, comparando-se as proporções pelo teste do qui-quadrado. Para o estudo das variáveis independentes associadas ao risco de nascimento com PI (não/sim) foram calculadas a Odds Ratio (OR) bruta e os intervalos de confiança, para cada ano de estudo. Posteriormente, foi realizada a análise multivariada, utilizando um modelo de regressão logística múltipla para testar o possível efeito gerador de confusão das variáveis independentes descritas anteriormente. O nível de significância adotado em todas as análises foi de 0,05.

O presente estudo foi aprovado pelo Comitê de Ética em Pesquisa do Hospital das Clínicas da Faculdade de Medicina de Ribeirão Preto da Universidade de São Paulo.

\section{Resultados}

Do total de 6.750 nascidos vivos em 1978/79, após a exclusão de 483 com baixo peso ao nascer e 42 com peso ignorado ao nascer, restaram 6.223 para análise. Em 1994, foram excluídos 303 com baixo peso e 21 com peso ignorado ao nascer, restando 2.522 nascidos vivos. Houve aumento significativo no número de nascimentos de crianças com PI de $1978 / 79$ para 1994 , de $22,4 \%(1.395 / 6.223)$ para $28,7 \%(724 / 2.522)(p<0,001)$.

A Tabela 1 compara a distribuição de características maternas, sociais, da gestação, parto e RN em Ribeirão Preto, em 1978/79 e 1994, nas crianças nascidas com peso igual ou superior a $2.500 \mathrm{~g}$. Não houve diferença entre as proporções de meninos e meninas entre os dois estudos $(p=0,734)$. A proporção de mães adolescentes ( $<20$ anos) aumentou e também a de mães mais velhas ( $\geq 30$ anos). A proporção de mães trabalhando fora de casa praticamente dobrou e o mesmo aconteceu com mães sem companheiro; a proporção de primíparas aumentou de $35 \%$ para $40,7 \%$, enquanto a de grandes multíparas ( $\geq 5$ filhos) reduziu-se à metade. $\mathrm{O}$ tabagismo materno diminuiu de $26,9 \%$ para $18,5 \%$. A freqüência de mães com escolaridade alta ( $\geq 12$ anos de estudo) aumentou em $30 \%$, enquanto a baixa escolaridade ( $\leq 4$ anos) reduziu-se a menos da metade. Foi também observada maior proporção de chefes de família em ocupações mais qualificadas e menor proporção em ocupações sem qualificação. Renda familiar baixa ( $\leq 3$ salários-mínimos) caiu em $1 / 3$, enquanto a renda mais alta ( $\geq 10$ salários-mínimos) aumentou de $10,6 \%$ para $16,1 \%$. Contudo, essa variável foi a que apresentou maiores proporções de informações ignoradas nos dois momentos (18,7 e 28,7\%, respectivamente). Não houve variação nas proporções de mães que referiram ter tido abortos prévios (17,5\% em 1978/79 e 16,4\% em 1994, $p=0,138)$ e natimortos prévios $(2,7 \%$ em $1978 / 79$ e $2,1 \%$ em 1994, $p=0,071$ ) entre os dois estudos.

Ainda na Tabela 1 , verifica-se que a atenção ao pré-natal praticamente universalizou-se: apenas $2,2 \%$ das mães não tiverem esse atendimento em 1994, contra $6,7 \%$ em 1978/79. Mais de 2/3 das mães no segundo estudo fizeram seis ou mais consultas, enquanto, no primeiro estudo, menos da metade o fez. A categoria particular de internação para o parto aumentou mais de três vezes e a participação dos hospitais públicos nesse atendimento reduziu-se quase à metade, enquanto a participação dos hospitais privados aumentou mais de oito vezes. A proporção de cesarianas apresentou um expressivo aumento no período, passando 
Tabela 1 - Características maternas, sociais, da gestação, do parto e do recém-nascido em Ribeirão Preto, 1978/79 e 1994

\begin{tabular}{|c|c|c|c|c|c|c|c|}
\hline Variáveis & $\begin{array}{c}1978 / 79 \\
N(\%)\end{array}$ & $\begin{array}{l}1994 \\
\text { N (\%) }\end{array}$ & $p$ & Variáveis & $\begin{array}{c}1978 / 79 \\
N(\%)\end{array}$ & $\begin{array}{l}1994 \\
\text { N (\%) }\end{array}$ & $p$ \\
\hline Idade materna & & & $<0,001$ & Situação conjugal & & & $<0,001$ \\
\hline$<20$ & $839(13,5)$ & $433(17,1)$ & & Com companheiro & $5.760(92,6)$ & $2.121(84,1)$ & \\
\hline $20 \mid-25$ & $2.044(32,8)$ & $736(29,2)$ & & Sem companheiro & $392(6,3)$ & $292(11,6)$ & \\
\hline $25 \mid-30$ & $1.804(29,0)$ & $684(27,1)$ & & Ignorado & $71(1,1)$ & $109(4,3)$ & \\
\hline $30 \mid-35$ & $966(15,5)$ & $436(17,3)$ & & Visitas no pré-natal & & & $<0,001$ \\
\hline$\geq 35$ & $510(8,2)$ & $226(9,0)$ & & 0 & $419(6,7)$ & $55(2,2)$ & \\
\hline Ignorado & $60(1,0)$ & $7(0,3)$ & & $1 \mid-4$ & $786(12,6)$ & $123(4,9)$ & \\
\hline Mãe trabalha fora do lar & & & $<0,001$ & $4 \mid-6$ & $1.290(20,7)$ & $276(10,9)$ & \\
\hline Não & $4.915(79,0)$ & $1.514(60,0)$ & & $\geq 6$ & $2.940(47,3)$ & $1.843(73,1)$ & \\
\hline Sim & $1.165(18,7)$ & $913(36,2)$ & & Ignorado & $788(12,7)$ & $225(8,9)$ & \\
\hline Ignorado & $143(2,3)$ & $95(3,8)$ & & Tipo de parto & & & $<0,001$ \\
\hline Número de filhos & & & $<0,001$ & Vaginal & $4.310(69,3)$ & $1.244(49,3)$ & \\
\hline 1 & $2.178(35,0)$ & $1.026(40,7)$ & & Cesárea & $1.913(30,7)$ & $1.278(50,7)$ & \\
\hline $2 \mid-5$ & $3.196(51,4)$ & $1.337(53,0)$ & & Internação & & & $<0,001$ \\
\hline$\geq 5$ & $666(10,7)$ & $131(5,2)$ & & Público & $5.532(88.9)$ & $1.486(58,9)$ & \\
\hline Ignorado & $183(2,9)$ & $28(1,1)$ & & Privado & $650(10,4)$ & $917(36,4)$ & \\
\hline Tabagismo materno & & & $<0,001$ & Ignorado & $41(0,7)$ & $119(4,7)$ & \\
\hline Não & $4.341(69,8)$ & $1.965(77,9)$ & & Renda familiar & & & $<0,001$ \\
\hline Sim & $1.676(26,9)$ & $465(18,5)$ & & $<3$ & $2.161(34,7)$ & $510(20,2)$ & \\
\hline Ignorado & $206(3,3)$ & $92(3,6)$ & & $3 \mid-5$ & $1.256(20,2)$ & $419(16,6)$ & \\
\hline Escolaridade mãe (anos) & & & $<0,001$ & $5 \mid-10$ & $986(15,8)$ & $464(18,4)$ & \\
\hline$\geq 12$ & $636(10,2)$ & $339(13,4)$ & & $\geq 10$ & $657(10,6)$ & $404(16,1)$ & \\
\hline $9 \mid-12$ & $827(13,3)$ & $549(21,8)$ & & Ignorado & $1.163(18,7)$ & $725(28,7)$ & \\
\hline $5 \mid-9$ & $1.534(24,7)$ & $930(36,9)$ & & Tipo de hospital & & & $<0,001$ \\
\hline$\leq 4$ & $3.052(49,0)$ & $531(21,0)$ & & Universitário & $942(15,1)$ & $472(18,7)$ & \\
\hline Ignorado & $174(2,8)$ & $173(6,9)$ & & Público & $5.022(80,7)$ & $1.126(44,6)$ & \\
\hline Ocupação chefe famíliar* & & & $<0,001$ & Privado & $259(4,2)$ & $924(36,6)$ & \\
\hline Não manual & $1.039(16,7)$ & $538(21,3)$ & & Pré-termo & & & $<0,001$ \\
\hline Manual Q/SQ & $3.541(56,9)$ & $1.404(55,7)$ & & Sim & $6.015(96,7)$ & $2.357(93,5)$ & \\
\hline Manual NQ/DESEMP & $1.237(19,9)$ & $457(18,1)$ & & Não & $208(3,3)$ & $165(6,5)$ & \\
\hline Ignorado & $406(6,5)$ & $123(4,9)$ & & Sexo do RN & & & 0,734 \\
\hline Adequação peso/IG** & & & 0,267 & Feminino & $2.996(48,1)$ & $1.225(48,6)$ & \\
\hline PIG & $443(7,2)$ & $193(7,7)$ & & Masculino & $3.221(51,8)$ & $1.296(51,4)$ & \\
\hline AIG & $5.410(86,9)$ & $2.199(87,2)$ & & Ignorado & $6(0,1)$ & $1(0,0)$ & \\
\hline GIG & $370(5,9)$ & $130(5,1)$ & & & & & \\
\hline
\end{tabular}

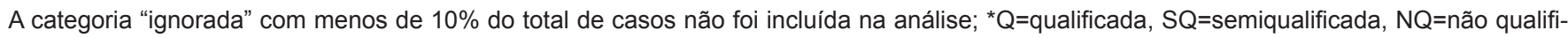
cado, DESEMP=desempregado; ${ }^{* *} \mathrm{IG}=$ idade gestacional, $\mathrm{PIG}=$ pequeno/IG, AIG=adequado/IG, GIG=grande/IG; ${ }^{* * *}$ Renda familiar em número de salários-mínimos por mês 
de $30,7 \%$ para $50,7 \%$. A proporção de $\mathrm{RN}$ pré-termo dobrou, embora não tenha repercutido na adequação do peso para a idade gestacional $(p=0,267)$.

As Tabelas 2 e 3 mostram as OR não ajustadas das variáveis associadas ao PI ao nascer em ao menos uma das coortes, para as características maternas (Tabela 2) e características socioeconômicas, da gestação, do parto e do RN (Tabela 3). Mães com menos de 20 anos e com menos de nove anos de escolaridade tiveram maior chance de ter filhos com PI $(p<0,001)$ e essas associações não se repetiram no ano de 1994 (Tabela 2). Trabalho materno fora do lar se associou com PI somente no ano de 1994 e hábito de fumar materno foi associado com PI nos dois estudos (Tabela 2). Abortos, natimortos prévios e número de filhos não apresentaram associação com PI ao nascer ( $p>0,05$ nas duas coortes).

Outras variáveis associadas ao PI no primeiro momento (1978/79) e que não se repetiram em 1994 foram (Tabela 3): menos de quatro visitas pré-natal $(p<0,001)$, categoria de internação pública $(p<0,001)$, grupo ocupacional manual não qualificado/desempregado $(p<0,001)$ e renda familiar menor que três salários-mínimos mensais $(p<0,001)$. Nascer em hospital universitário $(p<0,001)$ e de parto vaginal $(p<0,001)$ foram fatores associados à maior chance de ter PI em 1978/79; porém, tal associação foi fraca em 1994 $(p=0,050$ e $p=0,044$, respectivamente, para tipo de hospital e tipo de parto). Mães sem companheiro, bebês pré-termo e do sexo feminino tiveram maiores proporções de PI nos dois estudos.

Após ajustes para possíveis fatores geradores de confusão, a Tabela 4 mostra que, em 1978/79, a renda familiar acima de cinco salários-mínimos, a idade materna a partir de 20 anos, o sexo masculino, quatro ou mais visitas no pré-natal e o parto cesárea estiveram associados à menor chance de nascimento com PI. Filhos de mães fumantes e nascidos pré-termo tiveram risco cerca de duas vezes e mais de duas vezes e meia, respectivamente, de apresentar PI ao nascer. Observa-se que, na coorte de 1994, as variáveis socioeconômicas não mais se associaram ao PI ao nascer. Tabagismo materno, nascimento pré-termo e

Tabela 2 - Odds Ratio (OR) não ajustado e intervalo de confiança 95\% (IC95\%) para o peso insuficiente ao nascer segundo características maternas, em Ribeirão Preto, 1978/79 e 1994

\begin{tabular}{|c|c|c|c|c|c|c|}
\hline \multirow{2}{*}{ Variáveis } & \multicolumn{2}{|c|}{$1978 / 79$} & \multirow{2}{*}{$p$} & \multicolumn{2}{|c|}{1994} & \multirow{2}{*}{$p$} \\
\hline & $\mathbf{N}(\%)^{*}$ & OR (IC95\%) & & $\mathbf{N}(\%)^{*}$ & OR (IC95\%) & \\
\hline Idade & & & $<0,001$ & & & 0,910 \\
\hline$<20$ & $258(30,7)$ & 1,00 & & $125(28,9)$ & 1,00 & \\
\hline $20 \mid-25$ & $483(23,6)$ & $0,70(0,58-0,83)$ & & $214(29,1)$ & $1,01(0,77-1,31)$ & \\
\hline $25 \mid-30$ & $357(19,8)$ & $0,55(0,46-0,67)$ & & $197(28,8)$ & $0,99(0,76-1,30)$ & \\
\hline $30 \mid-35$ & $176(18,2)$ & $0,50(0,40-0,62)$ & & $118(27,1)$ & $0,91(0,67-1,23)$ & \\
\hline$\geq 35$ & $112(22,0)$ & $0,63(0,49-0,82)$ & & $69(30,5)$ & $1,08(0,76-1,54)$ & \\
\hline Ignorado & $9(15,0)$ & & & $1(14,3)$ & & \\
\hline Trabalho fora do lar & & & 0,084 & & & 0,012 \\
\hline Não & $1.128(23,0)$ & 1,00 & & $408(26,9)$ & 1,00 & \\
\hline Sim & $240(20,6)$ & $0,87(0,74-1,01)$ & & $289(31,7)$ & $1,25(1,05-1,50)$ & \\
\hline Ignorado & $27(18,9)$ & & & $27(28,1)$ & & \\
\hline Tabagismo & & & $<0,001$ & & & $<0,001$ \\
\hline Não & $821(18,9)$ & 1,00 & & $522(26,6)$ & 1,00 & \\
\hline Sim & $532(31,7)$ & $1,98(1,75-2,26)$ & & $176(37,8)$ & $1,68(1,36-2,08)$ & \\
\hline Ignorado & $42(20,4)$ & & & $26(28,3)$ & & \\
\hline Escolaridade (anos) & & & $<0,001$ & & & 0,250 \\
\hline$\geq 12$ & $100(15,7)$ & 1,00 & & $87(25,7)$ & 1,00 & \\
\hline $9 \mid-12$ & $160(19,4)$ & $1,28(0,97-1,69)$ & & $153(27,9)$ & $1,12(0,82-1,52)$ & \\
\hline $5 \mid-9$ & $344(22,4)$ & $1,55(1,21-2,00)$ & & $289(31,1)$ & $1,30(0,98-1,73)$ & \\
\hline$\leq 4$ & $754(24,7)$ & $1,76(1,40-2,21)$ & & $156(29,4)$ & $1,20(0,88-1,63)$ & \\
\hline Ignorado & $37(21,3)$ & & & $39(22,5)$ & & \\
\hline
\end{tabular}

A categoria "ignorada" com menos de $10 \%$ do total de casos não foi incluída na análise; *N(\%)=número e proporção do PI ao nascer em cada categoria das variáveis 
Tabela 3 - Odds Ratio (OR) não ajustado e intervalo de confiança 95\% (IC95\%) para o peso insuficiente ao nascer segundo características socioeconômicas, da gestação, parto e do recém-nascido, em Ribeirão Preto, 1978/79 e 1994

\begin{tabular}{|c|c|c|c|c|c|c|}
\hline \multirow{2}{*}{ Variáveis } & \multicolumn{2}{|c|}{$1978 / 79$} & \multirow[b]{2}{*}{$p$} & \multicolumn{2}{|c|}{1994} & \multirow{2}{*}{$p$} \\
\hline & $\mathbf{N}(\%)^{*}$ & OR (IC95\%) & & $\mathbf{N}(\%)^{*}$ & OR (IC95\%) & \\
\hline Grupo ocupacional & & & $<0,001$ & & & 0,297 \\
\hline Não manual & $187(18,0)$ & 1,00 & & $146(27,1)$ & 1,00 & \\
\hline Manual qualificado/semiqualificado & $781(22,1)$ & $1,29(1,08-1,54)$ & & $400(28,5)$ & $1,06(0,87-1,36)$ & \\
\hline Manual não qualificado/desempregado & $324(26,2)$ & $1,62(1,32-1,98)$ & & $144(31,5)$ & $1,23(0,94-1,62)$ & \\
\hline Ignorado & $103(25,4)$ & & & $34(27,6)$ & & \\
\hline Situação conjugal & & & $<0,001$ & & & 0,013 \\
\hline Com companheiro & $1.260(21,9)$ & 1,00 & & $592(27,9)$ & 1,00 & \\
\hline Sem companheiro & $124(31,6)$ & $1,65(1,32-2,06)$ & & $102(34,9)$ & $1,39(1,07-1,79)$ & \\
\hline Ignorado & $11(15,5)$ & & & $30(27,5)$ & & \\
\hline Número de visitas no pré-natal & & & $<0,001$ & & & 0,103 \\
\hline 0 & $130(31,0)$ & 1,00 & & $20(36,4)$ & 1,00 & \\
\hline $1 \mid-4$ & $230(29,3)$ & $0,92(0,71-1,19)$ & & $47(38,2)$ & $1,08(0,56-2,09)$ & \\
\hline $4 \mid-6$ & $269(20,8)$ & $0,59(0,46-0,75)$ & & $81(29,3)$ & $0,72(0,39-1,33)$ & \\
\hline$\geq 6$ & $580(19,7)$ & $0,55(0,43-0,68)$ & & $513(27,8)$ & $0,67(0,38-1,18)$ & \\
\hline Ignorado & $186(23,6)$ & $0,68(0,52-0,89)$ & & $63(28,0)$ & $0,68(0,37-1,27)$ & \\
\hline Tipo de parto & & & $<0,001$ & & & 0,044 \\
\hline Vaginal & $1.067(24,8)$ & 1,00 & & $380(30,6)$ & 1,00 & \\
\hline Cesárea & $328(17,2)$ & $0,63(0,55-0,72)$ & & $344(26,9)$ & $0,83(0,70-0,99)$ & \\
\hline Internação & & & $<0,001$ & & & 0,582 \\
\hline Público & $1.281(23,2)$ & 1,00 & & $432(29,1)$ & 1,00 & \\
\hline Privado & $108(16,6)$ & $0,66(0,53-0,82)$ & & $257(28,0)$ & $0,95(0,79-1,14)$ & \\
\hline Ignorado & $6(14,6)$ & & & $35(29,4)$ & & \\
\hline Renda familiar* & & & $<0,001$ & & & 0,612 \\
\hline$<3$ & $557(25,8)$ & 1,00 & & $132(25,9)$ & 1,00 & \\
\hline $3 \mid-5$ & $271(21,6)$ & $0,79(0,67-0,93)$ & & $125(29,8)$ & $1,21(0,92-1,62)$ & \\
\hline $5 \mid-10$ & $177(17,9)$ & $0,63(0,52-0,76)$ & & $134(28,9)$ & $1,16(0,88-1,54)$ & \\
\hline$\geq 10$ & $111(16,9)$ & $0,58(0,46-0,73)$ & & $117(29,0)$ & $1,17(0,87-1,56)$ & \\
\hline Ignorado & $279(24,0)$ & $0,90(0,77-1,07)$ & & $216(29,8)$ & $1,21(0,94-1,57)$ & \\
\hline Tipo de hospital & & & $<0,001$ & & & 0,05 \\
\hline Universitário & $270(28,7)$ & 1,00 & & $155(32,8)$ & 1,00 & \\
\hline Público & $1.084(21,6)$ & $0,68(0,58-0,79)$ & & $301(26,7)$ & $0,75(0,59-0,94)$ & \\
\hline Privado & $41(15,5)$ & $0,45(0,31-0,65)$ & & $268(29,0)$ & $0,83(0,65-1,06)$ & \\
\hline Pré-termo & & & $<0,001$ & & & $<0,001$ \\
\hline Não & $1.306(21,7)$ & 1,00 & & $648(27,5)$ & 1,00 & \\
\hline Sim & $89(42,8)$ & $2,69(2,03-3,57)$ & & $76(46,1)$ & $2,25(1,63-3,10)$ & \\
\hline Sexo do RN & & & $<0,001$ & & & $<0,001$ \\
\hline Feminino & $772(25,8)$ & 1,00 & & $420(34,3)$ & 1,00 & \\
\hline Masculino & $621(19,3)$ & $0,69(0,61-0,77)$ & & $304(23,5)$ & $0,58(0,79-0,70)$ & \\
\hline Ignorado & $2(0,1)$ & & & & & \\
\hline
\end{tabular}

A categoria "ignorada" com menos de $10 \%$ do total de casos não foi incluída na análise; *N(\%)=número e proporção do $\mathrm{PI}$ ao nascer em cada categoria das variáveis 
RN do sexo feminino mantiveram a associação observada em 1978/79. Trabalho materno fora do lar apresentou associação fraca ao PI. O tipo de parto não se associou ao PI em 1994, como observado na primeira coorte.

\section{Discussão}

A principal motivação deste estudo foi o aumento expressivo de nascidos com PI observado entre as coortes de 1978/79 e 1994, de 22,4 para 28,7\%. Em Campinas, São Paulo, a participação do PI foi grande, porém mantida em torno de $24 \%$ nos nascimentos da cidade no período de 1975 a $1996^{(16)}$, assim como as taxas de baixo peso ao nascer. Os autores consideram que a melhoria nos indicadores sociais e demográficos de Campinas identificados na década anterior ao estudo foi suficiente apenas para estabilizar a distribuição do peso ao nascer. Estudo realizado em 1991, em nove maternidades paulistas, mostrou que o PI foi observado em $19 \%$ dos RN doentes dessas maternidades ${ }^{(17)}$.

As variáveis socioeconômicas que mostraram associação com o PI em 1978/79, como renda familiar e número de visitas de pré-natal, assim como a idade materna (no modelo ajustado), não se mantiveram associadas em 1994. Isso pode ser justificado pela melhoria das condições

Tabela 4 - Modelo ajustado das variáveis associadas ao peso insuficiente ao nascer, em Ribeirão Preto, $1978 / 79$ e 1994

\begin{tabular}{|c|c|c|c|c|c|c|}
\hline \multirow{2}{*}{ Variáveis } & \multicolumn{3}{|c|}{$1978 / 79$} & \multicolumn{3}{|c|}{1994} \\
\hline & OR & IC $95 \%$ & $p$ & OR & IC95\% & $p$ \\
\hline Sexo do recém-nascido & & & $<0,001$ & & & $<0,001$ \\
\hline Feminino & 1,00 & & & 1,00 & & \\
\hline Masculino & 0,68 & $0,60-0,77$ & & 0,57 & $0,47-0,69$ & \\
\hline Tabagismo materno & & & $<0,001$ & & & $<0,001$ \\
\hline Não & 1,00 & & & 1,00 & & \\
\hline Sim & 1,94 & $1,69-2,21$ & & 1,62 & $1,29-2,04$ & \\
\hline Pré-termo & & & $<0,001$ & & & $<0,001$ \\
\hline Não & 1,00 & & & 1,00 & & \\
\hline Sim & 2,68 & $1,98-3,62$ & & 2,29 & $1,63-3,24$ & \\
\hline Mãe trabalha fora do lar & & & - & & & 0,020 \\
\hline Não & & & & 1,00 & & \\
\hline Sim & & & & 1,25 & $1,03-1,52$ & \\
\hline Renda familiar (SM) & & & 0,030 & & & - \\
\hline$<3$ & 1,00 & & & & & \\
\hline $3 \mid-5$ & 0,90 & $0,75-1,07$ & & & & \\
\hline $5 \mid-10$ & 0,76 & $0,62-0,94$ & & & & \\
\hline$\geq 10$ & 0,72 & $0,57-0,93$ & & & & \\
\hline Ignorado & 0,93 & $0,77-1,12$ & & & & \\
\hline Idade materna (anos) & & & 0,014 & & & - \\
\hline$<20$ & 1,00 & & & & & \\
\hline $20 \mid-25$ & 0,79 & $0,65-0,97$ & & & & \\
\hline $25 \mid-30$ & 0,73 & $0,59-0,89$ & & & & \\
\hline $30 \mid-35$ & 0,67 & $0,53-0,86$ & & & & \\
\hline$\geq 35$ & 0,82 & $0,62-1,08$ & & & & \\
\hline Visitas de pré-natal & & & 0,003 & & & - \\
\hline 0 & 1,00 & & & & & \\
\hline $1 \mid-4$ & 0,90 & $0,67-1,19$ & & & & \\
\hline $4 \mid-6$ & 0,67 & $0,51-0,88$ & & & & \\
\hline$\geq 6$ & 0,87 & $0,54-0,90$ & & & & \\
\hline Ignorado & 0,91 & $0,64-1,17$ & & & & \\
\hline Tipo de parto & & & $<0,001$ & & & - \\
\hline Vaginal & 1,00 & & & & & \\
\hline Cesárea & 0,71 & $0,61-0,82$ & & & & \\
\hline
\end{tabular}

$\mathrm{SM}=$ salários-mínimos 
gerais de vida e nível educacional da população, além do aumento do número de consultas de pré-natal observadas em Ribeirão Preto nesse período ${ }^{(18)}$.

Em 1978/79, o parto cesárea mostrou-se fator protetor contra o PI, o que não foi evidenciado em 1994. Parto cesárea foi o principal fator associado ao aumento das taxas de baixo peso em Ribeirão Preto, nesse intervalo de 15 anos, e também esteve associado à redução da média do peso ao nascer, bem como ao aumento dos nascimentos pré-termo ${ }^{(12,19)}$. Nesse período, houve aumento das taxas de cesariana de 30 para 50,1\%(20), proporções que se mantiveram mesmo após a exclusão dos RN de baixo peso da presente análise. Assim como em Ribeirão Preto, também em Campinas o parto operatório predominou sobre o vaginal e, mesmo que não tenha sido ainda identificado como fator de risco para o baixo peso ao nascer nessa cidade, pode ter modificado a freqüência de $\mathrm{RN}$ com $\mathrm{PI}^{(16)}$.

O trabalho materno fora do lar apresentou associação com PI ao nascer apenas em 1994. Isso pode ser explicado pelo grande influxo de mulheres no mercado de trabalho nas últimas décadas, o que também foi observado neste estudo. Acredita-se que essa associação ocorra principalmente entre mães com trabalho que exige atividade física mais intensa e que, neste caso, está também relacionado ao trabalho de parto pré-termo. Na Argentina, em 1984/85, o trabalho materno de mais de quatro horas por dia foi fator de risco para baixo peso ao nascer ${ }^{(21)}$. Em 1989, na Espanha, observou-se menor incidência de baixo peso de nascimento nos filhos de mães que trabalhavam fora ${ }^{(22)}$. Resultados semelhantes têm sido encontrados na maioria dos estudos em países desenvolvidos, onde as mulheres geralmente têm empregos sedentários. Contudo, o grande número de informações ignoradas nos dois estudos pode ter interferido nessa associação.

$\mathrm{Na}$ análise da influência do sexo do $\mathrm{RN}$ sobre o peso de nascimento, foi observada maior prevalência de PI em RN do sexo feminino nos dois momentos. Tal associação já foi demonstrada em diversos estudos e pode ser explicada, em parte, pela maior proporção de massa muscular em detrimento de tecido adiposo nos $\mathrm{RN}$ do sexo masculino, fato que lhes confere maior peso ao nascimento, enquanto o oposto ocorre nas meninas ${ }^{(23)}$. No estudo de Siqueira $e t a l^{(10)}$, dos nascidos com PI, 60,7\% eram do sexo feminino e 39,3\%, meninos. Contudo, neste estudo, a proporção de nascimentos de me- ninas foi semelhante nos dois momentos, não explicando, portanto, o aumento do PI no período.

O hábito materno de fumar é reconhecidamente fator de risco para o BP e PI ao nascer, além de outros efeitos nocivos ao concepto ${ }^{(1,9)}$. Rocha ${ }^{(1)}$ demonstrou que, dos filhos de mães tabagistas, 35,3\% nasciam com PI, enquanto essa fração entre filhos de não fumantes era de $27,8 \%$. No atual estudo, o tabagismo esteve associado ao PI nas duas coortes. Entretanto, neste intervalo de 15 anos, houve redução das taxas de tabagismo materno. Com isso, o hábito de fumar materno não estaria associado ao aumento da prevalência do PI neste período.

$\mathrm{O}$ contrário foi observado quanto à prevalência de nascimentos pré-termos, que, neste intervalo, aumentou de 7,6\% em 1978/79 para 13,6\% em 1994 ${ }^{(14)}$, considerando-se todos os nascimentos, e de 3,3 para 6,5\% após a exclusão das crianças nascidas de baixo peso. Considerando-se, então, que a prematuridade esteve associada com o PI nestes dois momentos, tal variável relaciona-se potencialmente ao aumento do PI ao nascer neste período. $\mathrm{Na}$ análise de Bettiol et al ${ }^{(14)}$, a elevação do número de nascimentos pré-termo, por sua vez, teve como maior contribuinte o aumento na frequiência de parto cesárea.

Os principais pontos positivos deste trabalho são: a mesma metodologia foi utilizada para a coleta de dados nos dois estudos, a taxa de participação foi quase completa e todas as maternidades participaram. A duração da gestação foi calculada com base na informação da data da última menstruação em ambas as coortes. Embora a variável principal em análise seja o PI ao nascer, o efeito adverso da perda de informação da idade gestacional foi minimizado por meio da utilização do método de imputação da idade gestacional. Apesar do grande número de fatores utilizados na análise, vários outros relacionados ao PI são encontrados na literatura e não estão presentes neste estudo, como doenças e infecções maternas, pouco ganho de peso na gestação, peso deficiente pré-gestacional, fatores genéticos, fatores placentários, uso de drogas, etilismo materno, nascimentos anteriores com PI, entre outros.

Esta análise sugere que o aumento do nascimento de crianças prematuras seja o maior responsável pelo incremento na prevalência de PI neste intervalo de 15 anos. Trabalho materno fora do lar, $\mathrm{RN}$ do sexo feminino e tabagismo materno durante a gravidez tiveram contribuição menos expressiva no fenômeno do aumento do PI ao nascer. 


\section{Referências bibliográficas}

1. Rocha JA. Baixo peso, peso insuficiente e peso adequado ao nascer em 5940 nascidos vivos na cidade de Recife: associação com algumas variáveis maternas. J Pediatr (Rio J) 1991;67:297-304.

2. Haeffner LSB, Barbieri MA, Rona RJ, Bettiol H, Silva AAM. The relative strength of weight and length at birth in contrast to social factors as determinants of height at 18 years in Brazil. Ann Hum Biol 2002;29:627-40.

3. Yamamoto RM, Leone CA. A influência das condições de vida no crescimento de lactentes nascidos com peso insuficiente. Rev Paul Pediat 2003;21:137-42.

4. Barker DJP, editor. Mothers, babies and health in later life. $2^{\text {nd }}$ ed. Edinburgh: Churchill Livingstone; 1998.

5. Moraes IB. Fatores de risco para peso insuficiente ao nascer [dissertação de mestrado]. Campinas (SP): Unicamp; 2001.

6. Barros FC, Victora CG, Vaughan JP, Estanislau HJ. Bajo peso al nascer en el municipio de Pelotas, Brasil: factores de riesgo. Bol Oficina Sanit Panam 1987;102:541-54.

7. Souza MLR, Tanaka ACA, Siqueira AAF, Santana RM. Estudo sobre nascidos vivos em maternidade. Rev Saude Publ 1988;22:489-93.

8. Puffer RR, Serrano CV. El peso al nascer, la edad materna y el orden de nacimiento: tres importantes factores determinantes de la mortalidad infantil, publicación científica 294. Washington: Organización Panamericana de la Salud, 1975.

9. Puffer RR, Serrano CV. Características del peso al nacer, publicación científica 504. Washington: Organización Panamericana de la Salud, 1988.

10. Siqueira AAF, Areno FB, Almeida PAM, Tanaka ACA. Relação entre peso ao nascer, sexo do recém-nascido e tipo de parto. Rev Saude Publ 1981;15:283-90.

11. Kramer MS. Determinants of low birth weight: methodological assessment and meta-analysis. Bull World Health Organ 1987;65:663-737.

12. Bettiol H, Rona RJ, Chinn S, Goldani M, Barbieri MA. Factors associated with preterm births in southeast Brazil: a comparison of two birth cohorts born 15 years apart. Pediatr Perinat Epidemiol 2000;14:30-8.
13. Barbieri MA, Gomes UA, Barros Filho AA, Bettiol H, Almeida LEA, Silva AAM. Saúde perinatal em Ribeirão Preto, SP, Brasil: a questão do método. Cad Saude Publ 1989;5:376-87.

14. Bettiol H, Barbieri MA, Gomes UA, Andrea M, Goldani MZ, Ribeiro ERO Saúde perinatal em Ribeirão Preto, SP, Brasil, 1994: metodologia e algumas características da população estudada. Rev Saude Publ 1998;32:18-28.

15. Rubin DB. Multiple imputation for nonresponse in surveys. New York: Wyley; 1987.

16. Mariotoni GGB, Barros Filho AA. Peso ao nascer e mortalidade hospitalar entre nascidos vivos, 1975-1996. Rev Saude Publ 2000;34:71-6.

17. GCEP (Grupo Colaborativo de Estudos Perinatais). Fatores perinatais relacionados com a morbidade e a mortalidade de recém-nascidos pertencentes a nove unidades neonatais do Município de São Paulo. J Pediatr (Rio J) 1996;72:379-87.

18. Goldani MZ, Bettiol H, Barbieri MA, Tomkins A. Maternal age, social changes and pregnancy outcome in Ribeirão Preto, southeast Brazil, in 1978-79 and 1994. Cad Saude Publ 2000;6:1041-7.

19. Silva AAM, Barbieri MA, Bettiol H, Goldani MZ, Rona RJ. Can we explain why Brazilian babies are becoming lighter? Int J Epidemiol 2004;33:821-8.

20. Silva AAM, Barbieri MA, Gomes UA, Bettiol H. Trends in low birth weight: a comparison of two birth cohorts separated by a 15-year interval in Ribeirão Preto, Brazil. Bull World Health Org 1998;76:73-84.

21. Belizán JM, Nardin JC, Carroli G, Campodónico L. Factores de riesgo de bajo peso al nascer em um grupo de embarazadas de Rosario, Argentina. Bol Oficina Sanit Panam 1989;106:380-7.

22. Rodríguez C, Regidor E, Gutiérrez-Fisac JL. Low birth weight in Spain associated with sociodemographic factors. J Epidemiol Community Health 1995;49:38-42.

23. Pinheiro CEA, Hornburg G, Batista FA. Epidemiologia perinatal da grande Florianópolis: II. Prevalência e análise do baixo peso ao nascer. Arq Catarin Med 1992;21:60-5. 